\title{
Molecular identification and sequencing of Pseudomonas aeruginosa virulence genes among different isolates in Al-Diwaneyah hospital
}

\author{
L.J. Shaebth \\ Al-Furat Al-Awsat Technical University, Technical Institute of Samawa, Email: ins.lyla@atu.edu.iq
}

(Received January 4, 2018; Accepted January 23, 2018)

\begin{abstract}
Pseudomonas $(P$.) aeruginosa possesses a variety of the virulence factors that may contribute to its pathogenicity, such as exotoxin A (toxA) and exoenzyme S (ExoS). The principal aim of this study was to find out the rapid method for identification of $P$. aeruginosa and to detect the toxA, exoS and 16SrRNA genes by Polymerase Chain Reaction (PCR) technique. Other aim on the other hand, the DNA sequencing was performed for phylogenetic tree analysis of 16SrRNA gene in local pathogenic $P$. aeruginosa isolates in comparison with NCBI-Genbank global $P$. aeruginosa isolates and finally submission of the present isolates in NCBI-Genbank database. According to the detection of the 16S rRNA gene, the study revealed that $29(58 \%)$ and $32(64 \%)$ of $P$. aeruginosa out of 50 swabs obtained from each wound and burn areas were positive. whereas in addition, the result of this study showed that the toxA gene was detected in $77 \%$ of $P$. aeruginosa isolated from the wound and $51 \%$ of $P$. aeruginosa isolated from the burn. whereas, the exoS gene was detected in $69 \%$ of $P$. aeruginosa isolated from the wound and $49 \%$ P. aeruginosa isolated from the burn. BLAST analysis showed that the $16 \mathrm{~S}$ rRNA gene shared more than $99 \%$ homology with the sequences of $P$. aeruginosa. Furthermore, the phylogenetic tree analysis of the 16S rRNA gene indicated that (PA$\mathrm{IQw}$ and PA-IQb) the 16S rRNA gene shared higher homology with other four $P$. aeruginosa isolates available in the GenBank. The homology of the nucleotides was between $99.9 \%$ and $100 \%$.
\end{abstract}

Keywords: Molecular identification, Pseudomonas uuiaeruginosa Available online at http://www.vetmedmosul.com

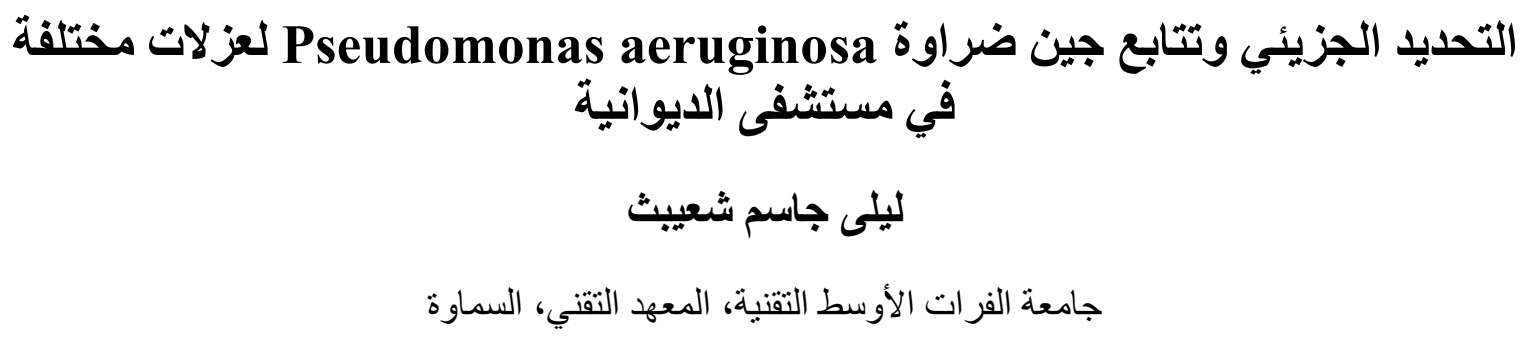

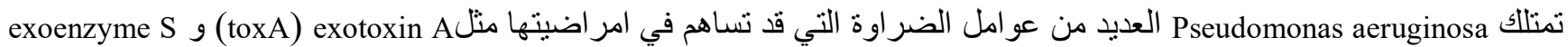

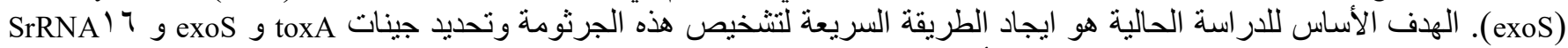

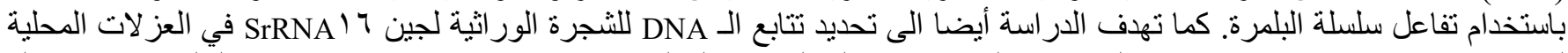

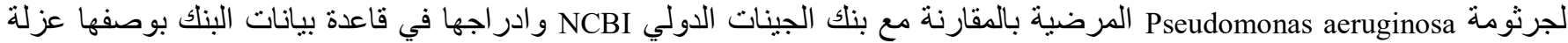

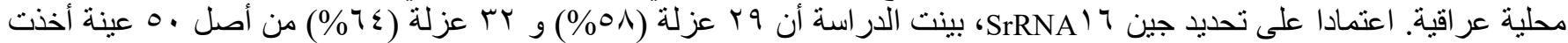

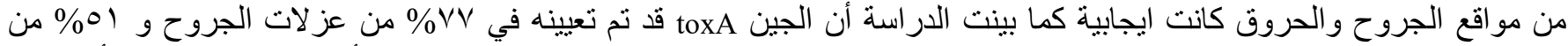

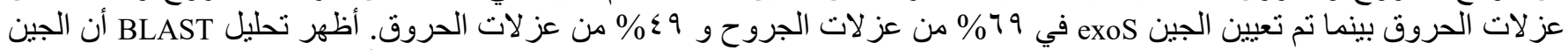

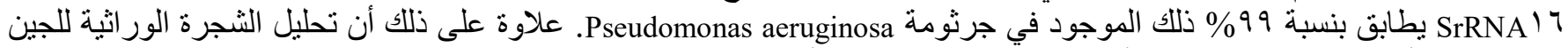

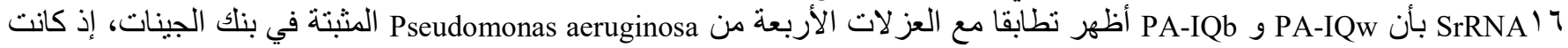

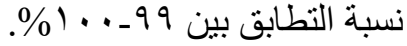




\section{Introduction}

$P$. aeruginosa is an opportunistic pathogen capable of infecting virtually all tissues. In addition, the $P$. aeruginosa has the ability to infect the immunocompromised individuals and responsible for hospital-acquired infections (1). Burn patients, mechanically ventilated patients, and cystic fibrosis (CF) patients are particularly susceptible to $P$. aeruginosa infections. It is a major cause of morbidity and mortality in patients with cystic fibrosis (2). $P$. aeruginosa infections in hospitals, mainly affect the patients in intensive care units and those having catheterization, burn, and/or chronic illnesses (3). $P$. aeruginosa possesses a variety of virulence factors that may contribute to its pathogenicity. $P$. aeruginosa also has a large number of the virulence factors such as exotoxin A, exoenzyme S, nan 1, and Las genes (1). The outer membrane proteins of $P$. aeruginosa are OprI and OprL that play the important roles in the interaction of the bacterium with the environment, as well as the inherent resistance of $P$. aeruginosa to the antibiotics. In addition, the presence of the specific outer membrane proteins have been implicated in the efflux transport systems that affect cell permeability (4).

As these proteins are found only in this organism, they could be a reliable factor for rapid identification of $P$. aeruginosa in clinical samples (5). $P$. aeruginosa can cause pulmonary damage by different mechanisms. Exoenzyme $\mathrm{S}$, encoded by the exoS gene, is an ADP ribosyltransferase that is secreted by a type-III secretion system directly into the cytosol of epithelial cells (6). Exotoxin A, encoded by the toxA gene, inhibits protein biosynthesis. The gene called nan 1 encodes a sialidase that is responsible for adherence to the respiratory tract (7). An extracellular neuraminidase is thought to play an important role in the implantation of the bacterium but the genetic basis of this process is still unknown (8). Although conventional microbiological methods for identifying $P$. aeruginosa from clinical and environmental samples are reliable, they require several days to be completed. Rapid detection of isolates which causing hospital infections is very important to take the final decision for treating the patients. PCR has the potential for identifying microbial species rapidly by amplification of sequences unique to a particular organism (9).

\section{Materials and methods}

\section{Sample collection}

One hundred swab samples were collected from wound and burn areas (50 for each) infection patients in AlDiwaneyah hospital. The samples were transported by sterile transport media then transferred into the microbiology laboratory of the College of Veterinary Medicine, University of Al-Qadisiyah, and kept at $4{ }^{\circ} \mathrm{C}$ until bacterial isolation.

\section{Bacterial isolation}

P. aeruginosa was isolated by inoculation on brain heart infusion (BHI) broth media and then incubated for overnight at $37^{\circ} \mathrm{C}$ for primary enrichment culture and then the growing of bacterial were inoculated on chrome agar and then incubated for overnight at $37^{\circ} \mathrm{C}$ for selecting the pure isolations of the $P$. aeruginosa.

\section{Bacterial genomic DNA extraction}

Bacterial genomic DNA was extracted from $P$. aeruginosa isolates by using PrestoTM Mini gDNA Bacteria Kit, Geneaid, USA. One $\mathrm{ml}$ of the growing of overnight bacterial growth on BHI broth was placed in 1.5 $\mathrm{ml}$ microcentrifuge tubes and then centrifuged at 10000 rpm for 1 minute. The supernatant was discarded and the bacterial cells pellets were used in genomic DNA extraction, and the extraction was done according to company instruction (Bioneer, Korea). The extracted gDNA was checked by Nanodrop spectrophotometer, then stored at $-20^{\circ} \mathrm{C}$ until PCR assay.

\section{Polymerase chain reaction (PCR)}

PCR assay was performed for detection based on $16 \mathrm{~S}$ rRNA gene and identification of genetic characterization of virulence factors genes for pathogenic P.aeruginosa using specific primers for detection of toxA and exoS virulence factors genes, according to method described by Nikbin et al. (8). These primers were designed by using the NCBIGenBank and the primer3 plus design online (Table 1).

Table 1: Oligonucleotide primers for amplification of various genes of P.aeruginosa

\begin{tabular}{|c|c|c|c|c|}
\hline Primer & & Sequence & Amplicon Size & GenBank code \\
\hline \multirow{2}{*}{ 16SrRNA } & $\mathrm{F}$ & 5-TGCCTGGTAGTGGGGGATAA-3 & \multirow{2}{*}{$505 \mathrm{bp}$} & \multirow{2}{*}{ MF067407.1 } \\
\hline & $\mathrm{R}$ & 5-GGATGCAGTTCCCAGGTTGA-3 & & \\
\hline \multirow{2}{*}{$\operatorname{exos}$} & $\mathrm{F}$ & 5-AGAGCGAGGTCAGCAGAGTA-3 & \multirow{2}{*}{$445 \mathrm{bp}$} & \multirow{2}{*}{ AY029251.1 } \\
\hline & $\mathrm{R}$ & 5-ATCCCGCTGACATCGATTCC-3 & & \\
\hline toxA & $\begin{array}{l}\mathrm{F} \\
\mathrm{R}\end{array}$ & $\begin{array}{l}\text { 5-CGTTCGCACATTCACCACTC-3 } \\
\text { 5-ACCATGGAGTAGTGCAGCAC-3 }\end{array}$ & $400 \mathrm{bp}$ & K01397.1 \\
\hline
\end{tabular}


The primers were provided by the Bioneer Company, Korea. The PCR master mix was prepared by using AccuPower ${ }^{\mathbb{B}}$ PCR PreMix kit, Bioneer, Korea. The PCR premix tube contains freeze-dried pellet of Taq DNA polymerase $1 \mathrm{U}$, dNTPs $250 \mu \mathrm{M}$, Tris- $\mathrm{HCl}(\mathrm{pH} 9.0) 10 \mathrm{mM}$, $\mathrm{KCl} 30 \mathrm{mM}, \mathrm{MgCl} 21.5 \mathrm{mM}$, stabilizer, and tracking dye. The PCR master mix reaction was prepared according to kit instructions in $20 \mu 1$ total volume by adding $5 \mu 1$ of purified genomic DNA and $1.5 \mu 1$ of 10 pmole of forward primer and $1.5 \mu \mathrm{l}$ of 10 pmole of reverse primer, then completing the PCR premix tube by deionizer PCR water into $20 \mu \mathrm{l}$ and briefly mixed by Exispin vortex centrifuge (Bioneer, Korea). The reaction was performed in a thermocycler (Bioneer, Korea) by set up the following thermocycler conditions; initial denaturation temperature of $95{ }^{\circ} \mathrm{C}$ for 5 min; followed by 30 cycles at denaturation $95{ }^{\circ} \mathrm{C}$ for $30 \mathrm{~s}$, annealing $58{ }^{\circ} \mathrm{C}$ for $30 \mathrm{~s}$, and extension $72{ }^{\circ} \mathrm{C}$ for $1 \mathrm{~min}$ and then final extension at $72{ }^{\circ} \mathrm{C}$ for $10 \mathrm{~min}$. The PCR products were examined by electrophoresis in a $1.5 \%$ agarose gel, stained with ethidium bromide, and visualized under UV transilluminator.

\section{DNA sequencing method}

The DNA sequencing method was performed for phylogenetic tree analysis of the 16S rRNA gene in the local pathogenic $P$. aeruginosa isolates and compared with NCBI-Genbank global $P$. aeruginosa isolates and final submission of the present isolates in NCBI-Genbank database. The 16S rRNA gene was purified from agarose gel by using EZ-10 Spin column DNA gel extraction kit (Biobasic, Canada). The purified 16S rRNA gene was sent to Macrogen Company in Korea for performing the DNA sequencing by using the $\mathrm{AB}$ DNA sequencing system. The phylogenetic tree analysis was performed based on the NCBI-Blast alignment identification and neighbor distance phylogenetic tree analysis (Mega version 6).

\section{Results}

The results of this study showed that the number of the $P$. aeruginosa isolated from the wound was 29 (58\%), while other samples from wound were negative. In addition, the results of this study showed that the number of the $P$. aeruginosa isolated from the burn was $32(64 \%)$, while other samples from burn were negative (Table 2).

Table 2: $P$. aeruginosa isolation results of transport media swabs from wound and burn infections

\begin{tabular}{lccc}
\hline Sample & Total samples & Positive isolate & Percent $\%$ \\
\hline Wound & 50 & 29 & 58 \\
Burn & 50 & 32 & 64 \\
\hline
\end{tabular}

By using PCR detection, all the $P$. aeruginosa isolates were positive which based on the 16S rRNA gene (figures 1-3). In the present study, the tox $A$ gene detected in the wound was $77 \%$ of isolates, and in the burn was $51 \%$ of isolates. While, the exoS gene detected in the wound was $69 \%$ of isolates and in the burn was isolates and burn was $49 \%$ of isolates (Table 3 ).

Table 3: Detection the toxA and $\operatorname{exoS}$ gene in the $P$. aeruginosa isolates from wound and burn infections

\begin{tabular}{lcc}
\hline $\begin{array}{l}\text { Virulence } \\
\text { gene }\end{array}$ & $\begin{array}{c}\text { P. aeruginosa isolates } \\
\text { from wound infection } \\
\text { total percent } \%\end{array}$ & $\begin{array}{c}\text { P. aeruginosa isolates } \\
\text { from burn infection } \\
\text { total percent } \%\end{array}$ \\
\hline ToxA & 77 & 51 \\
exoS & 69 & 49 \\
\hline
\end{tabular}

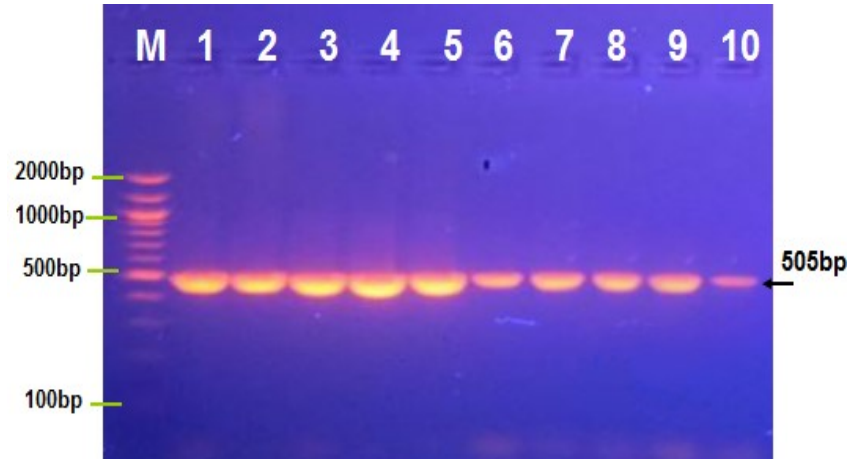

Figure 1: Agarose gel electrophoresis (1.5\%) showing the typical amplicon of the gene $16 \mathrm{~S}$ rRNA product of $P$. aeruginosa isolates. The amplification of DNA appears as a ladder-like pattern.A Where, Lane (M) DNA marker (100 bp), Lane (1-10) represent positive isolates.

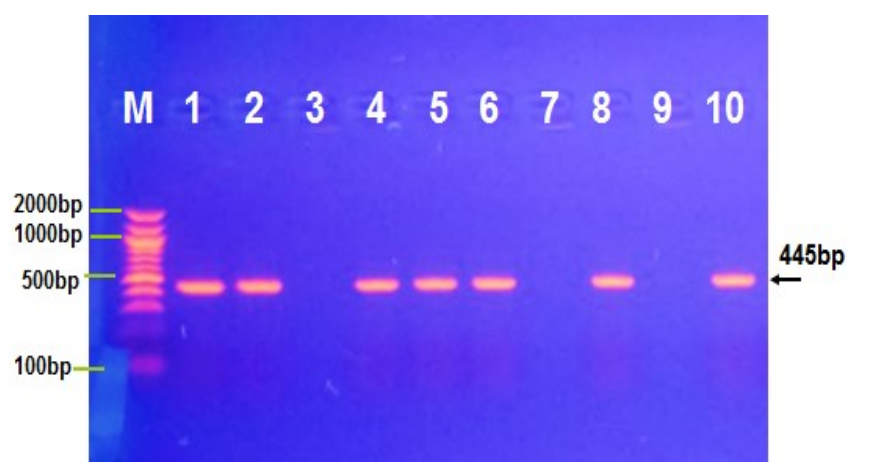

Figure 2: Agarose gel electrophoresis (1.5\%) showing the typical amplicon of the gene exoS product of $P$. aeruginosa isolates. The amplification of DNA appears as a ladder-like pattern.. Where, Lane (M) DNA marker (100 bp), Lane (1, $2,4,5,6,8$, and 10) represent positive isolates. Lane $(3,7$, and 9) represent negative isolates. 


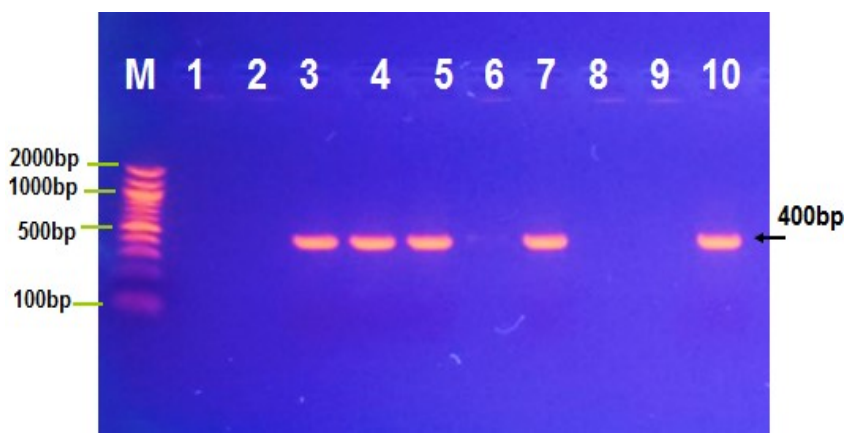

Figure 3: Agarose gel electrophoresis (1.5\%) showing the typical amplicon of the gene ToxA product of $P$. aeruginosa isolates. The amplification of DNA appears as a ladder-like pattern. Where, Lane (M) DNA marker (100 bp), Lane 3, 4, 5,7 , and 10) represent positive isolates. Lane $(1,2,6,8$, and 9) represents negative isolates.

\section{DNA sequencing results}

The DNA sequencing of the 16S rRNA gene that amplified by using PCR that was submitted in GenBank accession numbers MG062746 and MG062747. The BLAST analysis was showed that the $16 \mathrm{~S}$ rRNA gene shared more than $99 \%$ homology with the sequences of $P$. aeruginosa. Furthermore, the phylogenetic tree analysis was indicated that the 16S rRNA gene shared higher homology with the four $P$. aeruginosa isolates available in the GenBank. The homology of nucleotides was between $99.9 \%$ to $100 \%$ (figure 4 and 5 ).

\section{DNASequences Translated Protein Sequences \\ Species/Abbrv \\ 1. Pseudomonas aeruginosa wound isola GTCCGGAAACGGCCGCIAATACCGCATACGTCCTGAGGGAGAAAGTCGG \\ 2. Pseudomonas aeruginosa burn isolat GTCCGGAAACGGCCGCIAATACCGCATACGICCIGAGGGAGAAAGTCGG \\ 3. KR063148.1 Pseudomonas aeruginosa GTCCGGAAACGGCCGCTAATACCGCATACGTCCTGAGGGAGA A A TCGG \\ 4. KT962920.1 Pseudomonas aeruginosa GICCGGAAACGGCCGCTAATACCGCAIACGICCIGAGGGAGAAAGTCGG

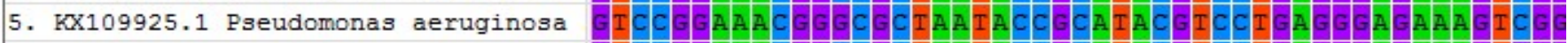 \\ 6. KX109926.1 Pseudomonas aeruginosa GTCCGGAAACGGCCGCTAATACCGCATACGICCIGAGGGAGAAAGIGGG \\ 7. KX447673.1 Pseudomonas aeruginosa GTCCGGAAACGGGCGCIAATACCGCA TACGICCIGAGGGAGA A ATGGG \\ 8. KX610179.1 Pseudomonas aeruginosa GICCGGAAACGGCCGCIAATACCGCATACGICCIGAGGGAGAAAGICGG \\ 9. KY412444.1 Pseudomonas aeruginosa GICCGGAAACGGGCGCTAATACCGCATACGICCTGAGGGAGAAAGTGGG

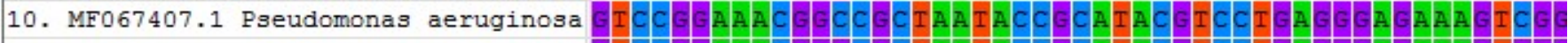 \\ 11. MG273769.1 Pseudomonas aeruginosaGTCCGGAAACGGGCGCTAATACCGCATACGTCCTGAGGGAGAAAGTGGG}

Figure 4: Multiple sequence alignment analysis of the partial 16SrRNA gene sequence in local $P$. aeruginosa wound and burn isolates (PA-IQw\& PA-IQb) based ClustalW alignment analysis by using (MEGA 6.0, multiple alignment analysis tool). That show the multiple alignment analysis similarity $\left(^{*}\right)$ and differences in $16 \mathrm{~S}$ rRNA gene nucleotide sequences.

\section{Discussion}

$P$. aeruginosa is a widely spread of the germ in the worldwide. The present study was carried out to isolate the $P$. aeruginosa from the two areas of the most important patients (wounds and burns) where 100 samples were collected from patients divided by 50 samples of wounds and 50 samples of burns and were cultured and isolated. The genetic material for all samples were extracted and diagnosed by using PCR. The virulence factor genes (ToxA and exoS) were recorded in the $P$. aeruginosa isolated from wounds more than of the $P$. aeruginosa isolated from the burns.

Identification of $P$. aeruginosa has traditionally relied on the phenotypic methods. This style is the most accurate standard when dealing with typical isolates of $P$. aeruginosa. The $P$. aeruginosa isolates display unusual phenotypic reactions. Moreover, biochemical testing takes a long time to perform and requires extensive hands on work by the technologist, both for setup and for on-going evaluation. The molecular methods have been reported to be superior to the phenotypic methods for for identifying of $P$. aeruginosa (10). De Vos et al. (5), by designing a multiplex PCR assay based on 16S rRNA gene for molecular detection of $P$. aeruginosa, that showed the specificity and sensitivity of the PCR assay were $100 \%$ (4). Lavenir et al. (11) also noted that all the P. aeruginosa strains contained the genes oprI and oprL (sensitivity = $100 \%$, specificity $=80 \%$ ). Similarly in this study, all of the isolates were remarkably positive for both oprI and oprL genes. According to these studies, detection of $P$. aeruginosa by PCR of oprI and oprL genes has a high 
sensitivity but a low specificity. The reason of low specificity of oprI and oprL genes is that, although the entire genome of $P$. aeruginosa has been sequenced, the genomes of its closest relatives have not. Thus, presence of false positive results, among other species of bacteria during PCR assay of oprI and oprL genes indicates that they may have some similar sequences to oprI and oprL genes in their genomes (10). Consequently, the use of only single gene target for molecular identification of $P$. aeruginosa potentially suffers from the same polymorphisms that complicate biochemical identification of this organism. PCR procedure was developed to detect the $P$. aeruginosa by amplifying the toxA gene (3). They reported that of 130 tested the P.aeruginosa isolates, 125 $(96 \%)$ contained the tox $A$ gene (sensitivity $=96 \%)$, whereas other species of bacteria did not yield any positive results (specificity $=100 \%$ ). Qin et al. (10) and Lavenir et al. (11) also reported similar results. These studies indicate that, unlike oprI and oprL genes, detection of $P$. aeruginosa by using PCR based on the tox $A$ gene has a high specificity but a low sensitivity $(10,11)$. In this study, the results also showed that $29(77 \%)$ of 50 isolates harbored toxA gene. The $p t x R$ gene, expression enhancer of toxA gene, was only detected in $P$. aeruginosa isolates; whereas other species of Pseudomonas did not yield any positive results (12). Low sensitivity with toxA PCR screening is due to the fact that some isolates of P.aeruginosa do not carry this gene naturally. Pathogenicity of $P$. aeruginosa is clearly multifactorial. The $P$. aeruginosa isolates are generally expressed the cytotoxicity or invasion phenotypes which correlated with presence of exoU (encoding exotoxin $\mathrm{U}$ ) or exoS (encoding exotoxin S) (13). In our study, there is a difference between exoS prevalence in the $P$. aeruginosa isolates from wound and in the $P$. aeruginosa isolates from burn infections. In this study, the proportion of isolates from wound infections that exhibited exoS was lower than that previously reported (10,14-16). The conflicting results of these studies may be due to differences in the number of clinical isolates from different sites or due to the isolates from patients with different clinical and physiological conditions $(14,15)$. About the nanl gene, the other virulence factor studied in this research, we found that the prevalence of nanl was significantly higher in isolates from wound than isolates from burn. Furthermore, the prevalence of nanl among the isolates from wound was significantly higher than burn.

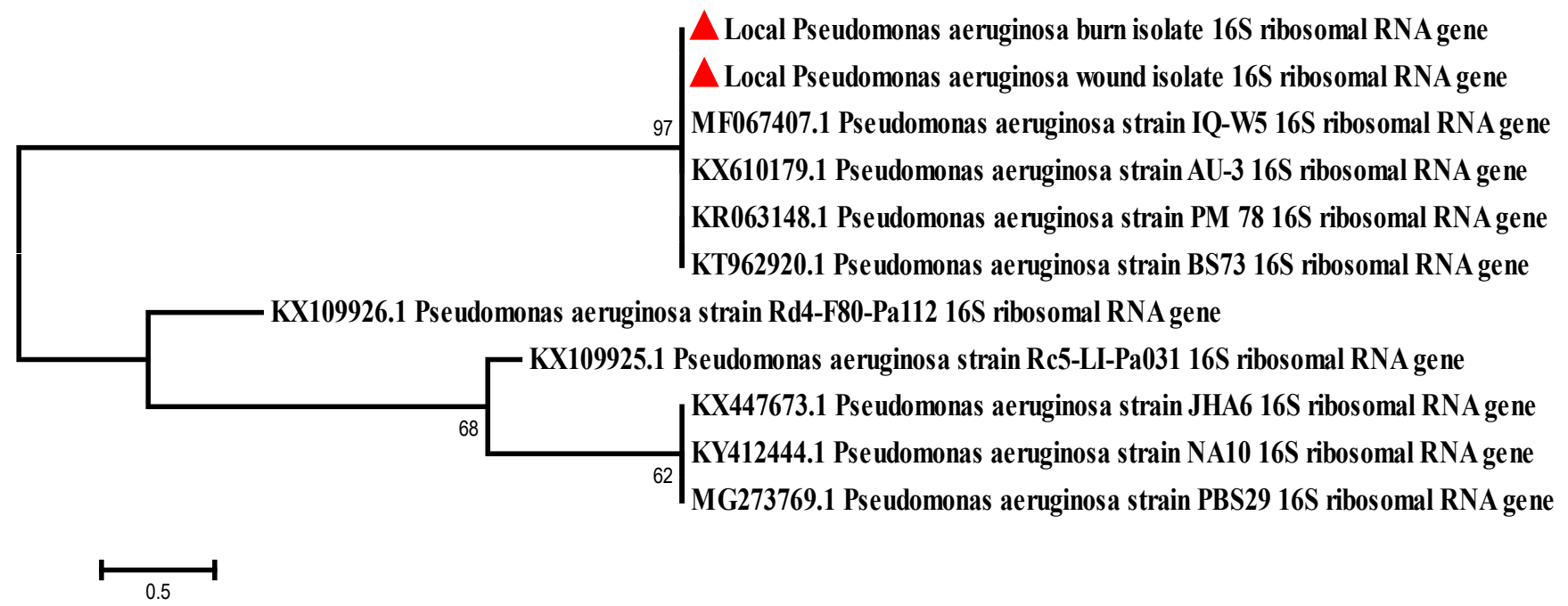

Figure 5: Phylogenetic tree analysis based on the 16S rRNA gene partial sequence that used for local $P$. aeruginosa wound and burn isolates (PA-IQw\& PA-IQb). The phylogenetic tree was constructed using Maximum Likelihood tree method in (MEGA 6.0 version). The local $P$. aeruginosa isolates PA-IQw\& PA-IQb were show closed related to NCBI-Blast $P$. aeruginosa strains (MF067407.1, KX610179.1, KR063148.1, and KT962920.1) with genetic change 0.5\%.

The low prevalence of this factor among isolates from burn infections may show that the role of this gene in the burn infections is less important than wound infections. The differences in the distributions of virulence factor genes in the populations strengthen the probability that some $P$. aeruginosa strains are better adapted to the specific conditions found in specific infectious sites (10).

In conclusion, this study is regarded as the first study of differentiation between two types of infections area by $P$. aeruginosa. It seems that simultaneous use of oprI, oprL and toxA genes provides more confident detection of $P$. 
aeruginosa by PCR. Determination of different virulence genes of $P$. aeruginosa isolates suggests that they are associated with different levels of the intrinsic virulence and pathogenicity. This may have different consequence on the outcome of infections. Significant correlations between some virulence genes and source of infections obtained in this research indicates that more further studies is required for finding out the actual role of these genes in different clinical infectious caused by $P$. aeruginosa. Ribotyping showed that strains with similar virulence genes do not necessarily have similar ribotype patterns. However, clonal spread of highly virulent isolates of $P$. aeruginosa within hospitals needs to apply additional precautions in clinical settings.

\section{References}

1. Van Delden C, Iglewski BH. Cell-to-cell signaling and Pseudomonas aeruginosa infections. Emerg Infect Dis. 1998;4:551-560.

2. Fegan M, Francis P, Hayward AC, Davis GH, Fuerst JA. Phenotypic conversion of Pseudomonas aeruginosa in cystic fibrosis. J Clin Microbiol. 1990;28:1143-1146.

3. Yetkin G, Otlu B, Cicek A, Kuzucu C, Durmaz R. Clinical, microbiologic, and epidemiologic characteristics of Pseudomonas aeruginosa infections in a university hospital, Malatya, Turkey. Am J Infect Control. 2006;34:188-192.

4. Nikaido H. Prevention of drug access to bacterial targets: Permeability barriers and active efflux. Sci. 1994;264:382-388.

5. De Vos D, Lim A, Pirnay JP, Struelens M, Vandenveld C. Direct detection and identification of Pseudomonas aeruginosa in clinical samples such as skin biopsy specimens and expectorations by multiplex PCR based on two outer membrane genes, oprI and oprL. J Clin Microbiol. 1997;35:1295-1299.
6. Yahr TL, Hovey AK, Kulich SM, Frank DW. Transcriptional analysis of the Pseudomonas aeruginosa exoenzyme $\mathrm{S}$ structural gene. J Bacteriol. 1995;177:1169-1178.

7. Bryan R, Kube D, Perez A, Davis P, Prince A. Overproduction of the CFTR R domain leads to increased levels of asialo GM1 and increased Pseudomonas aeruginosa binding by epithelial cells. Am J Respir Cell Mol Biol. 1998;19:269-277.

8. Nikbin VS, Aslani MM, Sharafi Z, Hashemipour M, Shahcheraghi F. Molecular identification and detection of virulence genes among Pseudomonas aeruginosa isolated from different infectious origins. Iran J Microbiol. 2012;4:118-123.

9. Lanotte P, Mereghetti L, Dartiguelongue N, Rastegar- Lari A, Gouden A. Genetic features of Pseudomonas aeruginosa isolates cystic fibrosis patients compared with those of isolates from other origins. $\mathrm{J}$ Med Microbiol. 2004;53:73-81.

10. Qin X, Emerson J, Stapp J, Stapp L, Abe P, Burns L. Use of real-time PCR with multiple targets to identify Pseudomonas aeruginosa and other nonfermenting gram-negative bacilli from patients with cystic fibrosis. J Clin Microbiol. 2003; 4:4312-4317.

11. Lavenir R, Jocktane D, Laurent F, Nazaret S, Cournoyer B. Improved reliability of Pseudomons aeruginosa PCR detection by the use of the specific ecfx gene target. J Microbiol Methods. 2007;70:20-29.

12. Vasil ML, Chamberlain C, Grant CCR. Molecular studies of Pseudomonas exotoxin A gene. Infect Immun 1986; 52:538-548.

13. Lomholt JA, Poulsen K, Kilian M. Epidemic population structure of Pseudomons aeruginosa: evidence for a clone that is pathogenic to the eye and that has a distinct combination of virulence factor. Infect Immun. 2001;69:6284-6295.

14. Winstanley C, Kaye SB, Neal TJ, Miksch S, Hart CA. Genotypic and phenotypic characterization of Pseudomonas aeruginosa isolates associated with ulcerative keratitis. J Med Microbiol. 2005;54:519526.

15. Feltman H, Schulert G, Khan S, Jain M, Peterson L, Hauser AR, Prevalence of type III secretion genes in clinical and environmental isolates of Pseudomonas aeruginosa. Microbiol. 2001;147:2659-2669.

16. Schulert GS, Feltman H, Rabin SDP, Martin CG, Battle SE, Rello J, et al. Secretion of the toxin ExoU is a marker for highly virulent Pseudomonas aeruginosa isolates obtained from patients with hospital-acquired pneumonia. J Infect Dis. 2003;188:1695-1706. 